\title{
CSB affected on the sensitivity of lung cancer cells to platinum-based drugs through the global decrease of let-7 and miR-29
}

\author{
Zhenbang Yang ${ }^{1,2}$, Chunling Liư ${ }^{3}$, Hongjiao Wu' ${ }^{1}$ Y Yuning Xie ${ }^{1,4}$, Hui Gao ${ }^{1,4}$ and Xuemei Zhang ${ }^{1 *}$ (D
}

\begin{abstract}
Background: Transcription-coupled nucleotide excision repair (TC-NER) plays a prominent role in the removal of DNA adducts induced by platinum-based chemotherapy reagents. Cockayne syndrome protein B (CSB), the master sensor of $T C R$, is also involved in the platinum resistant. Let-7 and miR-29 binding sites are highly conserved in the proximal 3'UTR of CSB.

Methods: We conducted immunohistochemisty to examine the expression of CSB in NSCLC. To determine whether let-7 family and miR-29 family directly interact with the putative target sites in the 3'UTR of CSB, we used luciferase reporter gene analysis. To detect the sensitivity of non-small cell lung cancer (NSCLC) cells to platinum-based drugs, CCK analysis and apoptosis analysis were performed.

Results: We found that let-7 and miR-29 negatively regulate the expression of CSB by directly targeting to the $3^{\prime} U T R$ of CSB. The endogenous CSB expression could be suppressed by let-7 and miR-29 in lung cancer cells. The suppression of CSB activity by endogenous let-7 and miR-29 can be robustly reversed by their sponges. Down-regulation of CSB induced apoptosis and increased the sensitivity of NSCLC cells to cisplatin and carboplatin drugs. Let-7 and miR-29 directly effect on cisplatin and carboplatin sensitivity in NSCLC.
\end{abstract}

Conclusions: In conclusion, the platinum-based drug resistant of lung cancer cells may involve in the regulation of let-7 and miR-29 to CSB.

Keywords: CSB, Let-7, miR-29, Platinum, Lung cancer

\section{Background}

Lung cancer is the leading cause of cancer-related death worldwide. Despite improvements in diagnosis and surgical techniques, platinum-based chemotherapy remains the foundation of treatment for lung cancer, in particular for patients with NSCLC; however, the efficacy is significantly limited. Multiple mechanisms have been causally linked to the platinum drug resistance, such as drug transport, drug detoxification, DNA repair and cell apoptotic [1-3]. To date, the organizing principles of platinum drug resistance are still not fully understood.

\footnotetext{
* Correspondence: jyxuemei@gmail.com

${ }^{1}$ Institute of Molecular Genetics, College of Life Science, North China

University of Science and Technology, Tangshan, China

Full list of author information is available at the end of the article
}

Platinum drugs (mainly cisplatin and carboplatin) form several types of DNA adduct lesions including the predominating 1,2-d(GpG) and 1,2-d(ApG) intrastrand crosslinks (90\%), followed by 1,3-d(GpNpG) intrastrand crosslinks (5$10 \%)$, with minor amounts of $1,2-\mathrm{d}(\mathrm{GpC})$ interstrand and DNA-protein crosslinks (2-5\%) [4]. The platinum-DNA intrastrand crosslinks are mainly repaired by NER [5], which also contributes to the removal of platinum-DNA interstrand adducts [6]. NER involves recognition and dual incision of the damage, followed by gap filling [7]. Various reports have convincingly shown that abnormal expression of key genes in the process of NER are highly correlated with platinum drug resistance in a variety of tumor types, particularly testicular, ovarian and NSCLC [8]. For example, ERCC1 is the most promising marker of resistance to

(c) The Author(s). 2019 Open Access This article is distributed under the terms of the Creative Commons Attribution 4.0 International License (http://creativecommons.org/licenses/by/4.0/), which permits unrestricted use, distribution, and reproduction in any medium, provided you give appropriate credit to the original author(s) and the source, provide a link to the Creative Commons license, and indicate if changes were made. The Creative Commons Public Domain Dedication waiver (http://creativecommons.org/publicdomain/zero/1.0/) applies to the data made available in this article, unless otherwise stated. 
cisplatin-based adjuvant therapy and the down-regulation of ERCC1-XPF by siRNA sensitizes lung cancer cells to cisplatin [9].

CSB, the master sensor of TC-NER, is overexpressed in a variety of cancers including lung cancer [10]. CSB plays a prominent role in the removal of both cisplatinDNA intrastrand and interstrand adducts $[6,11]$. Intriguingly, CSB has a large 4337-nucleotide-long 3'untranslated region (UTR), nearly half the length of the messenger RNA, which contains two perfectly conserved miRNA binding sites (let-7 and miR-29) among land vertebrates. Let-7, the well-known tumour suppressor family, is among the most abundantly expressed miRNAs in lung tissue. Global down-regulation of let-7 members is common in lung cancer tissue [12]. The miR-29 family is also down-regulated in lung cancer tissue and the re-expression of miR-29 in lung cancer cells can inhibit tumorigenesis [13]. Due to the evidences that these miRNAs are involved in the lung cancer, it is important to reveal the role of these miRNAs-driven pathway in the process of lung cancer.

In this study, we demonstrated that CSB is overexpressed in NSCLC tissue. We also found that let-7 and miR-29 directly target CSB and regulate the expression of CSB. Furthermore, our data showed that inactivation of CSB could induce apoptosis and increase the sensitivity of lung cancer cells to cisplatin and carboplatin. Our findings support a role for CSB adjuvant therapy as a viable strategy for cisplatin-based chemotherapeutic sensitivity in NSCLC.

\section{Methods}

\section{Immunohistochemistry}

Histopathological evaluation of human lung cancer was performed by experienced board-certified pathologists with HE-stained lung tumor sections. Patient samples were collected at Affiliated Tangshan Renmin Hospital (Tangshan, China). Their general characteristics were collected at the time of tumor sample collection, including gender, age and AJCC (TNM) tumor stage. This study was approved by the ethics committee of North China University of Science and Technology (No. 12-002). For CSB staining, 4- $\mu \mathrm{m}$ thickness sections cut on paraffinembedded lung tumor samples were deparaffinized, rehydrated and immersed in 3\% hydrogen peroxide solution for $10 \mathrm{~min}$ to quench endogenous peroxidase activity. After heat-induced antigen retrieval, tissues were blocked with 5\% BSA and incubated with CSB primary antibody (Abcam, ab96089) at 1:250. After that, sections were incubated with biotinylated anti-rabbit secondary antibody, avidin-biotin complex and then developed in $\mathrm{DAB}$ using a commercial detection kit (ZSGB-BIO, China, PV-8000) according to the manufacturer's instructions. Image acquisition was performed with Olympus BX63 microscope and a DP80 camera (Olympus). Quantification CSBpositive cells was performed by calculating $\mathrm{DAB}$ positive pixels per area and counted by an ImageJ script.

\section{Cell culture}

Human NSCLC cell lines A549, NCI-2030 and NCIH1975 were purchased from American Type Culture Collection (ATCC). A549 and NCI-H2030 cells were cultured in GibcoTM Roswell Park Memorial Institute 1640 (RPMI 1640) (Life Technologies, Grand Island, NY, USA) and NCI-H1975 cells were cultured in Dulbecco's Modified Eagle Medium (DMEM). All mediums were supplemented with $10 \%$ fetal bovine serum (FBS; Life Technologies, Grand Island, NY, USA) and antibiotics $(100 \mathrm{U} / \mathrm{ml}$ penicillin and $100 \mu \mathrm{g} / \mathrm{ml}$ streptomycin) in a humidified incubator with $5 \% \mathrm{CO}_{2}$ at $37^{\circ} \mathrm{C}$.

\section{Vector cloning}

The 3'UTR of CSB was amplified using primers 5' -CAC CTC GAG ACA ACA TTG CTT CCT AAA CTT TCA AG-3' (XhoI) and 5'-GTA AGC GGC CGC ACT AAG ACA GCT AAG AAG AAA T-3' (NotI). PCR product was subcloned into the psiCHECK2 reporter vector (Promega, Madison, MI, USA) to generate psiCHECK2CSB-3'UTR (WT construct). The let-7 or miR-29 binding site in the 3'UTR of CSB on WT construct were mutated by PCR based enzyme synthesis commercially (Synbio Technologies, China) to create MT-let-7 and MT-miR-29 constructs, respectively. The CMV-d2eGFPcxcr4 vector (Addgene plasmid 21,967) was digested with XhoI and PmeI and ligated a sponge insert (synthesized commercially, Synbio Technologies) containing 10× let-7, miR-29 and CXCR4 bulged binding sites (let7: AAC TAT ACA AAA CCT ACC TCA, miR-29: TAA CCG ATT TTC TTG GTG CTA, CXCR4: AAG TTT TCA GAA AGC TAA CA, 4 nt-spacer: CCGG) together to generate let-7, miR-29 and CXCR4 sponge.

\section{SiRNA and miRNA mimics transfection}

Lung cancer cells were seeded into 6-well or 96- well plates and transfected with $20 \mathrm{nM}$ siRNA or miRNA mimics (GenePharma, China) using Lipofectamine RNAiMAX Transfection Reagent (ThermoFisher Scientific, Grand Island, NY, USA) according to the manufacturer's instructions. Cells were harvested after $48 \mathrm{~h}$ for further analysis. The sequence of siRNA and miRNA are listed below. CSB siRNA (siCSB), 5'-GUG UGC AUG UGU CUU ACG A-3' (sense); Let-7a mimic, 5'-UGA GGU AGU AGG UUG UAU AGU U-3' (sense); let-7f mimic, 5'-UGA GGU AGU AGA UUG UAU AGU U-3' (sense); miR-29a mimic, 5'-UAG CAC CAU CUG AAA UCG GUU A-3' (sense); miR-29b mimic, 5'-UAG CAC CAU UUG AAA UCA GUG UU-3' (sense); miR-29c mimic, 5'-UAG CAC CAU UUG AAA UCG GUU A-3' 
(sense); control mimic, 5'-UUC UCC GAA CGU GUC ACG UTT-3' (sense).

\section{Luciferase assays}

To detect the binding of let-7/miR-29 with CSB 3'UTR, psiCHECK2 vector (WT construct, MT-let-7 or MTmiR-29 construct) was transfected into lung cancer cells using Lipofectamine 2000. We also co-transfected WT construct with either let-7 sponge, miR-29 sponge or CXCR4 sponge to A549 cells. Luciferase activities were determined using the Dual-Luciferase Assay System (Promega, Madison, MI, USA) according to the manufacturer's instructions. The GloMax20/20 Luminometer (Promega, USA) was used to measure fluorescence intensity, followed by a 2-s pre-read delay and a 10-s measurement period. Renilla luciferase activity was used to normalize firefly luciferase activity.

\section{Western blotting}

Western blot analysis was performed using standard methods. CSB protein levels were quantified with whole cell extracts using antibodies against CSB (Abcam, ab96089) and $\beta$-actin (Santa Cruz Biotechnology, sc-8342).

\section{qPCR}

Total RNA was isolated from lung cancer cells using Trizol reagent (ThermoFisher Scientific, NY, USA). For both mRNA and miRNA expression analysis, cDNA was prepared from $1 \mu \mathrm{g}$ RNA using Maxima $\mathrm{H}$ Minus First Strand cDNA Synthesis Kit with dsDNase (ThermoFisher Scientific, NY, USA) and oligo $(\mathrm{dT})_{18}$ primer. 20 ng of cDNA was then used for qPCR with the Power SYBR Green PCR Master Mix (ThermoFisher Scientific, NY, USA). The qPCR primers for targeting distinct polyadenylation sites on CSB 3'UTR and apoptosis analysis were list in Additional file 1. Relative expression was determined using the $2^{-\Delta \Delta C T}$ method.

\section{Generation of stable cell lines}

GV248 short hairpin RNA (shRNA) constructs were synthesized by Genechem (Shanghai, China). The sequence of CSB shRNA and control shRNA are $5^{\prime}$-GTG TGC ATG TGT CTT ACG A-3' and 5'-TTC TCC GAA CGT GTC ACG T-3', respectively. GV369 primiRNA expression constructs (Genechem, Shanghai, China): let-7f-1 pri-miRNA (forward: 5'-GAG GAT CCC CGG GTA CCG GTT TCT TTC GAA AGA GAT TGT ACT TTC CAT TC-3'; reverse: 5'-CAC ACA TTC CAC AGG CTA GTA CTT GAA CAG AGA AAA TTA AC-3'); miR-29a pri-miRNA (forward: 5'-GAG GAT CCC CGG GTA CCG GTC ATT CCA TTG TGC CTG G-3'; reverse: 5'-CAC ACA TTC CAC AGG CTA GTT GCT TTG CAT TTG TTT TC-3'); control vector (no insert). Lentiviral particles were produced by co- transfection of HEK293 cells with GV248/GV369 vectors and packaging vectors pHelper 1.0 and pHelper 2.0 from Shanghai Genechem. H2030 cells were infected with either shRNA or pri-miRNA lentivirus in 6-well plates and subsequently split into $10-\mathrm{cm}$ dishes $48 \mathrm{~h}$ after infection in the presence of $2 \mu \mathrm{g} / \mathrm{ml}$ puromycin for selection over 1 week.

\section{Drug treatment and CCK analysis}

For CCK analysis, lentiviral H2030 cells were treated with either 10-70 $\mu \mathrm{M}$ cisplatin (Sigma-Aldrich, USA) or $80-640 \mu \mathrm{g} / \mathrm{ml}$ carboplatin (Sigma-Aldrich, USA) for 24h. siRNA and miRNA mimics transfected H2030 cells were treated with either 4-32 $\mu \mathrm{M}$ cisplatin or 30$240 \mu \mathrm{g} / \mathrm{ml}$ carboplatin for $48 \mathrm{~h}$. Four thousand cells per well were plated on a 96-well plate in sextuplicate overnight. After drug treatment, cells were then treated with WST-8 Cell Counting reagent (Dojindo, Japan) for $1 \mathrm{~h}$ at $37^{\circ} \mathrm{C}$ according to the manufacturer's protocol. Analysis was performed using the Infinite M200 PRO Microplate Reader (Tecan) with $450 \mathrm{~nm}$ absorbance and the survival rate was calculated by normalizing untreated cells to $100 \%$.

\section{Apoptosis analysis}

PE Annexin V Apoptosis detection kit (BD Biosciences, CA) was used to detect apoptosis. Cells were treated with cisplatin $(12 \mu \mathrm{M})$ or carboplatin $(80 \mu \mathrm{g} / \mathrm{ml})$ for $48 \mathrm{~h}$ and were collected by centrifugation, resuspended in $400 \mu \mathrm{l}$ binding buffer, followed by staining with $5 \mu \mathrm{l} \mathrm{PE}$ Annexin $\mathrm{V}$ and $5 \mu \mathrm{l} 7$-ADD for $15 \mathrm{~min}$ in dark at room temperature. Apoptotic cells were then evaluated by 7 ADD and Annexin V-positive cells on a fluorescenceactivated cell-sorting (FACS) flow cytometer (Beckman Coulter, CA).

\section{Bioinformatics and statistical analysis}

To determine the potential miRNAs binding to CSB 3' UTR sequence, we used two commonly online miRNA prediction programs: TargetScan (http://www.targetscan. org/) and miRanda (http://www.microrna.org). The expression of CSB was analyzed using The Cancer Genome Atlas (TCGA) data sets (https://portal.gdc.cancer. gov) and Gene Expression Profiling Interactive Analysis (GEPIA) (http://gepia.cancer-pku.cn/).

\section{Statistical testing}

Data are expressed as means \pm SD. Statistical significance was assessed by the Student's t-test. $P$ values less than 0.05 were considered significant. 


\section{Results}

\section{CSB expression is up-regulated in NSCLC}

To examine the expression of CSB in NSCLC, we performed immunohistochemistry in 43 lung adenocarcinoma (LUAD) samples and 43 squamous carcinoma (LUSC) samples, and their paired adjacent normal tissues. As shown in Fig. $1 \mathrm{a}$ and b, CSB was mainly localized in the nucleus of lung cancer cells. In most lung cancer cases, we observed stronger staining of CSB than in normal tissues. The percentage of positive CSB in LUAD (72.56\%) and LUSC tissues (72.09\%) were significantly higher than that in paired adjacent normal tissues (19.61 and $18.96 \%$, respectively) $(P<0.001)$. We then analyzed the RNA expression of CSB in lung cancer and normal tissues using the GEPIA (483 LUAD vs 59 normal tissues; 486 LUSC vs 50 normal tissues). The RNA level of CSB in lung cancer tumor tissue was significantly higher than that in normal tissue (Fig. 1c).

Let-7 and miR-29 8mer binging sites are highly conserved in the proximal 3'UTR of CSB across species

Based upon the online miRNA target prediction tools, TargetScan and miRnada, two 8mer sites of let-7 (position 125-132) and miR-29 (position 367-374) are highlighted in the 3'UTR transcript of CSB (4337 nt, NM_000124), both residing in more proximal 3'UTR contexts (Fig. 2). Importantly, most of the 3'UTR of CSB is divergent in evolution among land vertebrates; however, the single 8 mer sites of let- 7 and miR-29 are broadly evolutionarily conserved across nearly all mammalian species.

An important consideration in the accurate prediction of miRNA-target interactions is the usage of alternative 3'UTR isoforms by influencing both the presence and scoring of target sites [14]. Some researchers have identified two alternative tandem 3' UTR isoforms of CSB (153 nt and $2160 \mathrm{nt}$ ) using Northern blot analysis [15]. Recently, longer tandem 3'UTR isoforms were found by sequencing (2370 nt, CR749388; 3449 nt, ENST00000355832; 4337 nt, NM_ 000124) (Fig. 3a, Additional file 1). Notably, each of these isoforms contains a canonical poly (A) signal (PAS) located in $35 \mathrm{nt}$ upstream of their corresponding poly (A) sites. Since the putative 8 mer site of let- 7 is located in the shortest 3'UTR isoform of CSB, all five identified 3'UTR transcripts are potentially subject to the let-7-mediated regulation. In contrast, the putative 8 mer site of miR-29 is located beyond the shortest one but in the second one, so the longer isoforms of CSB may affect the potential regulation by miR-29. In present study, we examined the relative

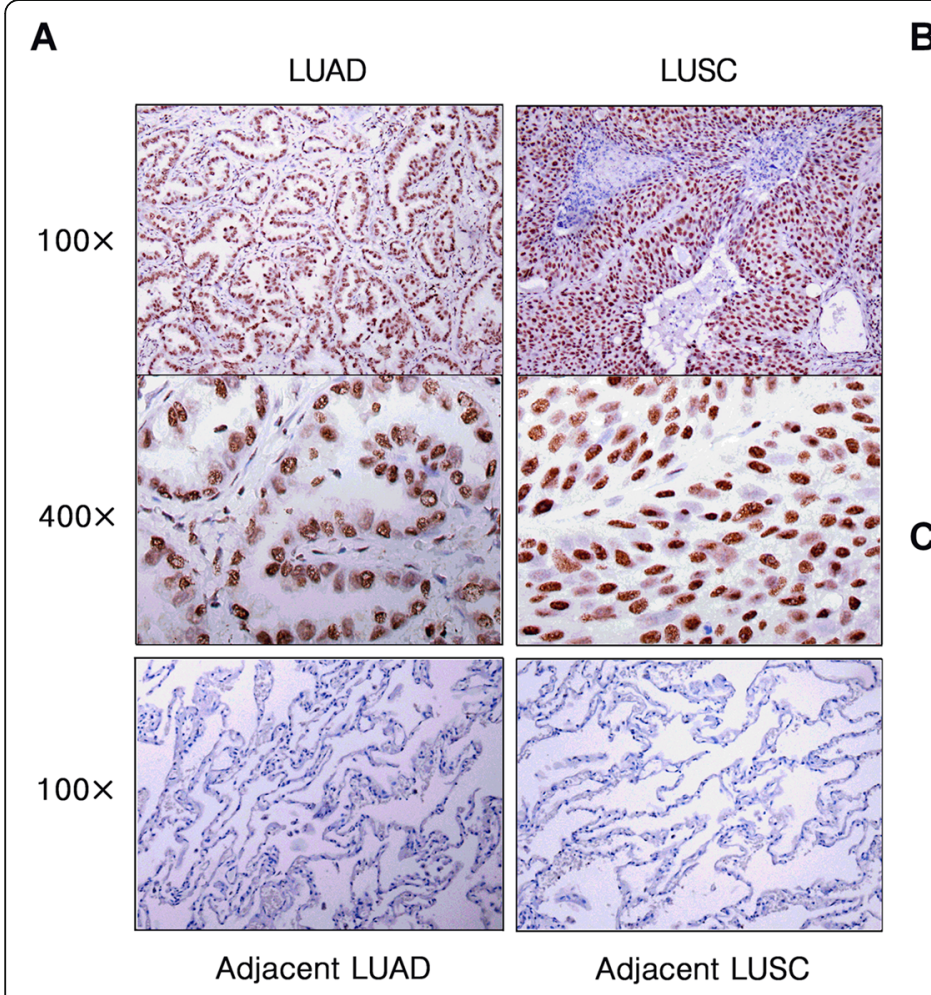

B

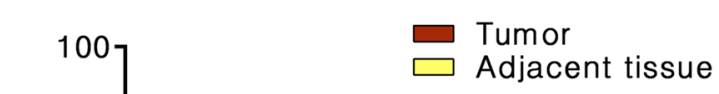

Fig. 1 Immunohistochemical visualization of CSB protein in NSCLC and adjacent lung tissues. a Representative CSB immunohistochemical staining of human NSCLC and normal lung tissues. b Percentage CSB positive LUAD and adjacent normal tissues ( $n=43$ ); LUSC and adjacent normal tissues $(n=43)$. LUAD, lung adenocarcinoma; LUSC, lung squamous carcinoma. c The CSB mRNA level in 483 LUAD tissues and 59 normal tissues, 486 LUSC tissues and 50 normal tissues from GEPIA 


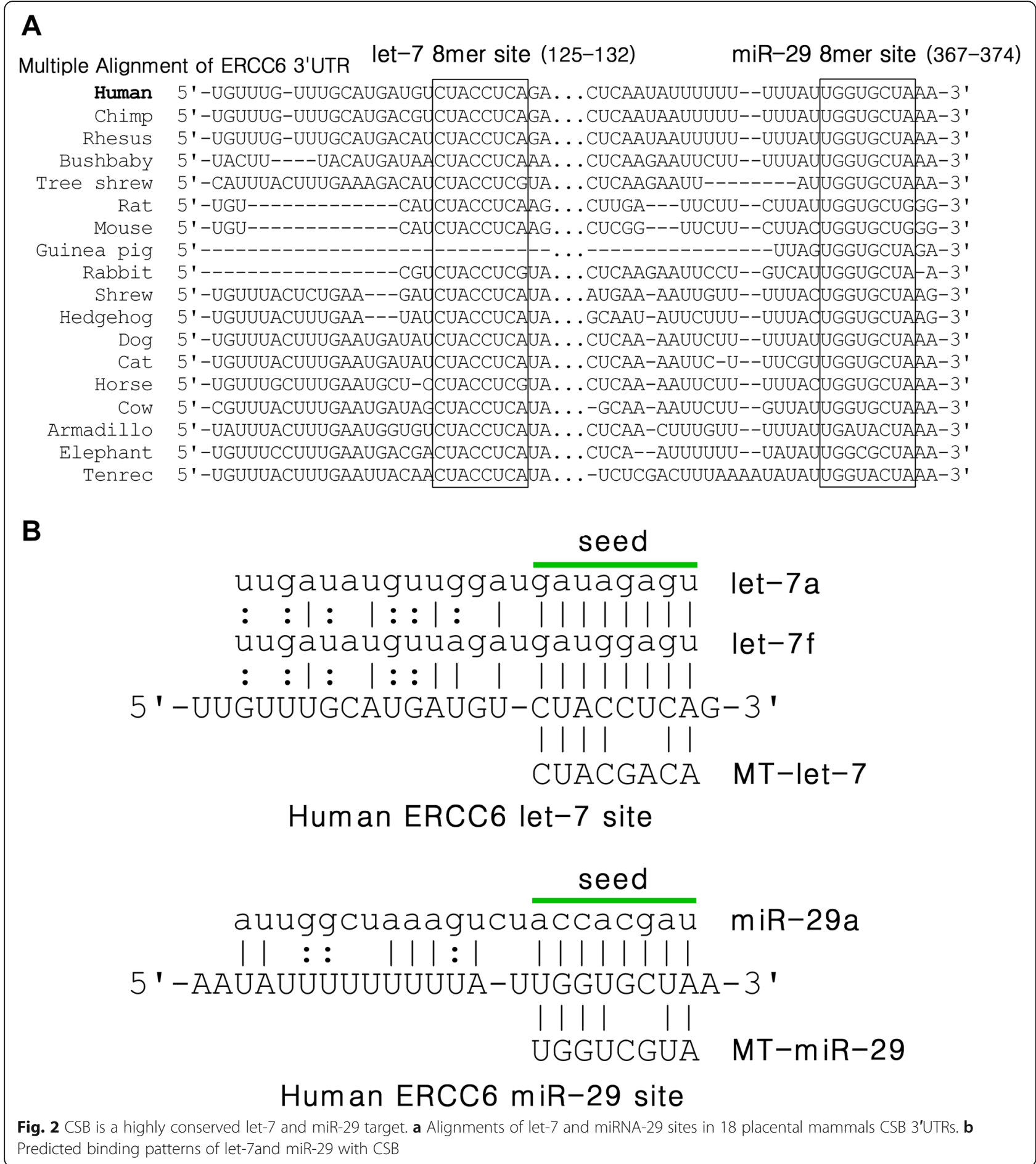

levels of these 3'UTR isoforms in lung cancer cells by specific qPCR analysis and observed a similar CSB expression in all transcript isoforms in A549, H1975, and H2030 cells (Fig. 3b). This result indicates that the longest known 4337 nt 3'UTR isoform might be prevalent in lung cancer cells and is also affected by the potential let-7 or miR-29 mediated regulation.
Let-7 and miR-29 directly target to the 3'UTR of CSB

To determine whether let-7 family and miR-29 family directly interact with the putative target sites in the 3' UTR of CSB, we generated three psiCHECK2-CSB-3' UTR luciferase reporter constructs with WT, MT-let-7 or MT-miR-29. We then co-transfected each construct with either let-7/miR-29 mimics or scrambled mimics 


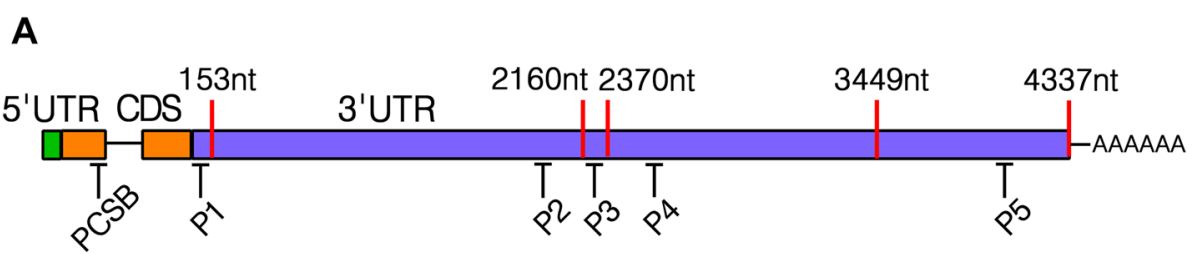

B

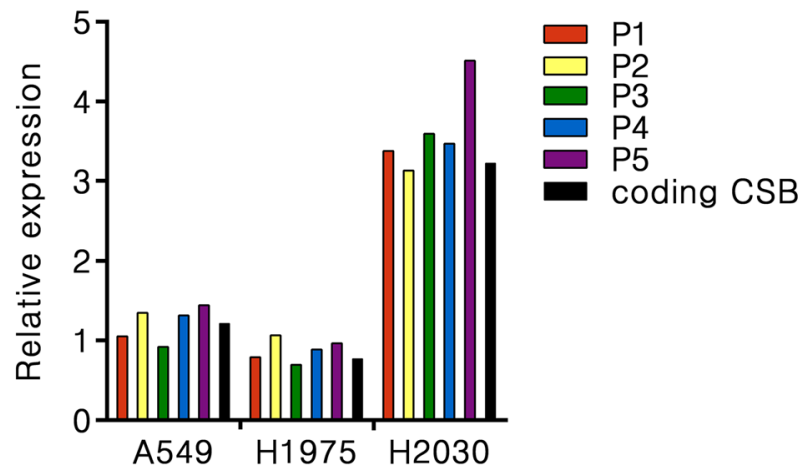

Fig. 3 ERCC6 gene expresses the longest known CSB-3'UTR isoform in NSCLC. a Schematic of human CSB 3'UTR-APA (alternative polyadenylation), indicating poly(A) sites (red strip) and their approximate location. Specific qPCR strategy amplifying five distinct CSB 3'UTR isoforms used in P1-P5 primer pairs. b qPCR analysis of distinct CSB 3'UTR isoforms levels in A549, H1975 and H2030 cells. Values are normalized to GAPDH. Mean of two independent experiments shown

into A549 cells. Representative let-7a and let-7f miRNAs highly expressed in A549 cells were used for further analysis [16]. Luciferase reporter gene analysis revealed that the relative Renilla luciferase activity of WT was reduced about $55 \%$ in response to additional let-7a or let-7f $(P<0.001)$ and $40 \%$ in response to additional miR-29a, $19 \%$ to miR-29b, $28 \%$ to miR-29c $(P<$ 0.01 ), whereas both MT-let-7 and MT-miR-29 showed no change of luciferase activity (Fig. 4).
Endogenous let-7 and miR-29 in lung cancer cells confirm the miRNA-mRNA interaction on CSB targets

As with the lung cancer cells highly expressing native let-7 and miR-29, we examined the interaction of endogenous let-7 and miR-29 with CSB targets by directly transfecting each psiCHECK2 construct into lung cancer cells (A549 and H1975). We observed a striking suppression of WT construct compared to MT-let-7/MT-miR29 in both cell lines $(P<0.01)$ (Fig. 5a).
A

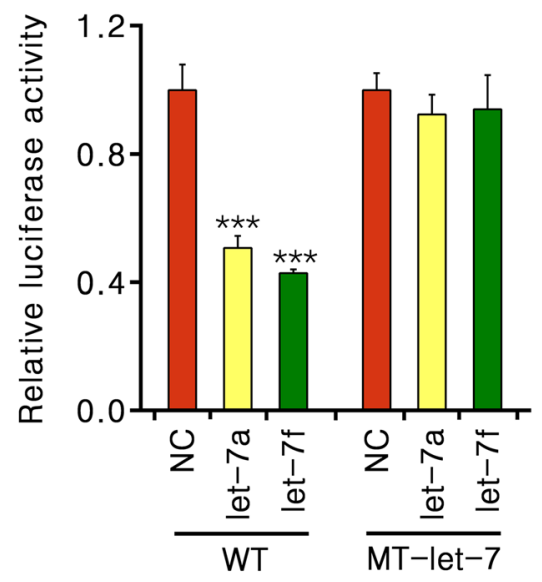

B

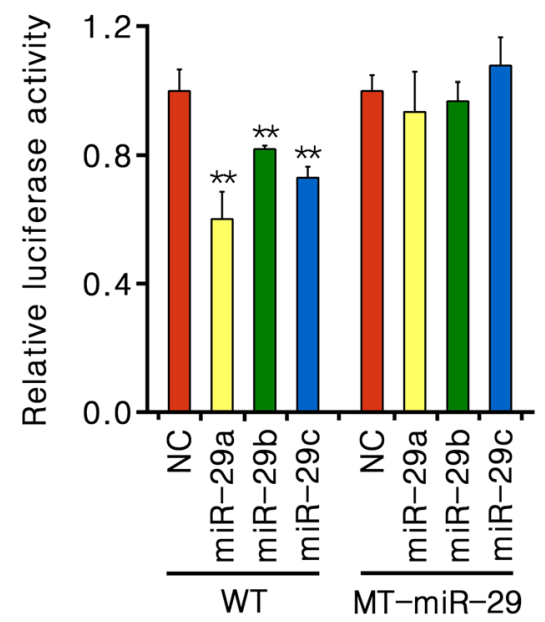

Fig. 4 Let-7 and miR-29 directly target to CSB 3'UTR. a and b Relative fluorescence ratio in A549 cells co-transfected with miRNA mimics and either the wild-type (WT) or let-7/miR-29-mutant CSB 3'UTR (MT-let-7/MT-miR-29) constructs. Values are normalized to NC miRNA mimic of either WT or MT-let-7/MT-miR-29 construct. ${ }^{*} P<0.01$, ${ }^{* *} P<0.001$ 
A

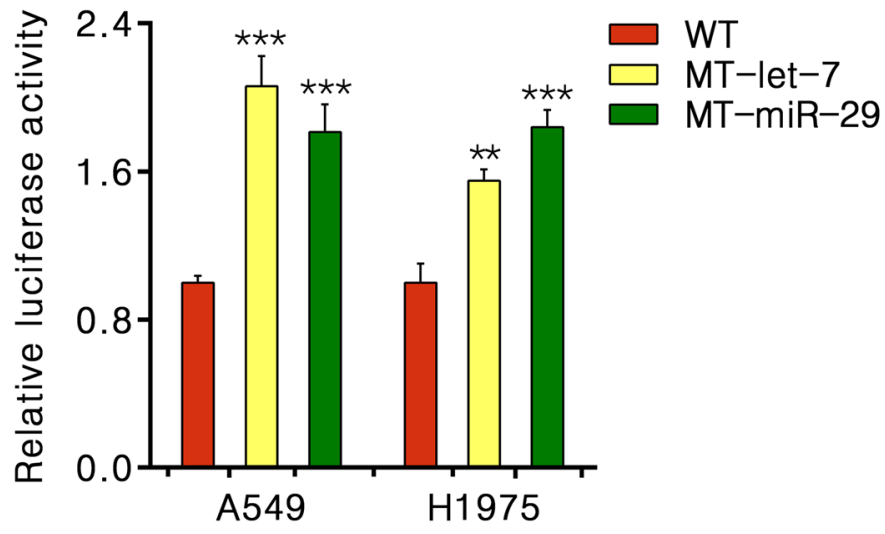

B

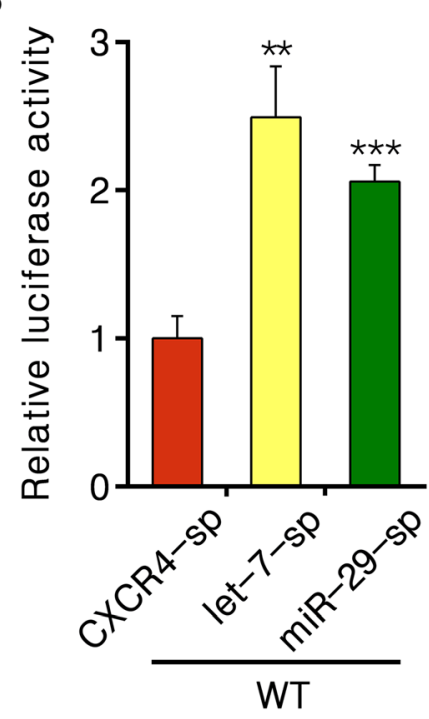

C

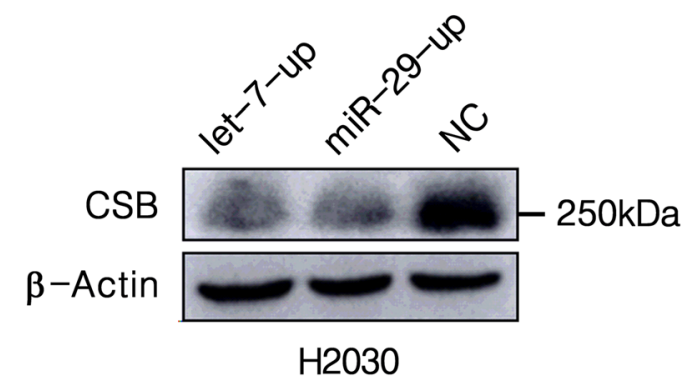

Fig. 5 CSB activity is regulated by endogenous let-7 and miR-29. a Relative fluorescence ratio in cells transfected with WT, MT-let-7 and MT-miR29 constructs respectively. b Relative fluorescence ratio in A549 cells co-transfected with WT and let-7 sponge (let-7-sp) miR-29 sponge (miR-29sp) or CXCR4 sponge (CXCR4-sp) constructs. Values are normalized to CXCR4-sp construct. ${ }^{* *} P<0.01,{ }^{* * *} P<0.001$. c Immunoblot for CSB in H2030 cells infected with lentiviral let-7f-1 and miR-29a pri-miRNAs

To elucidate the ability of endogenous let- 7 and miR-29 on CSB targets more broadly, we performed miRNA knockdown in A549 cell. Considering the presence of various let-7 and miR-29 isoforms in human lung cancer cells, we used miRNA sponges, which can function as sinks for pools of active miRNAs, liberating transcripts targeted by that set of miRNAs, to dilute all endogenous let-7 or miR-29 members in A549 cell specifically. As shown in Fig. 5b, the designed let-7 and miR-29 sponges efficiently blocked their function. The suppression of WT activity by endogenous let-7/miR-29 can be robustly reversed by let-7/miR-29 sponge $(P<0.01)$, but not by CXCR4 sponge.

\section{Increase in let-7 and miR-29 abundance in NSCLC cells} suppress endogenous CSB expression

To test the effect of let-7 and miR-29 on the expression of CSB, we generated lentiviral let-7f-1 and miR-29a pri-miRNA expression constructs and a control construct in which the pre-miRNA stem loop was deleted. After transfecting each construct into H2030 cells, we found that the corresponding expression of let- $7 f-1$ and miR-29a pri-miRNAs were increased over 100-fold compared to the control. As shown in Fig. 5c, CSB protein level was dramatically decreased with expression of either let-7f or miR-29a construct, suggesting the endogenous CSB expression can be suppressed by let-7 and miR-29. 
Down-regulation of CSB increases the sensitivity of NSCLC cells to cisplatin and carboplatin drugs

To assess the effect of CSB on the development of NSCLC treated by cisplatin or carboplatin, we transfected lentiviral short hairpin RNA (shRNA) construct to H2030 cells to knockdown CSB and observed a striking reduced CSB protein levels $(P<$ 0.001) (Fig. 6a). We then tested the cell viability after CSB knockdown in response to cisplatin and carboplatin. Significant growth suppression was observed in CSB-knockdown $\mathrm{H} 2030$ cells treated by cisplatin or carboplatin (Fig. 6b, c). In response to cisplatin and carboplatin, the 50\% inhibitory concentrations $\left(\mathrm{IC}_{50}\right)$ value was decreased 6-fold and 4-fold after inhibiting CSB. These results indicate that CSB promotes the formation of cisplatin and carboplatin resistance in NSCLC, with potential implications for lung cancer chemotherapy.
CSB inhibition induces apoptosis to sensitize platinum resistant NSCLC cells

To better understand the physiological role of CSB expression in NSCLC cells, we examined apoptosis activity in H2030 cells treated with CSB siRNA and control siRNA by Flow Cytometer. As shown in Fig. 7, the overexpression of CSB in lung cancer cells treated with cisplatin and carboplatin could induce apoptosis and the knockdown of CSB by siRNA significantly increased apoptosis.

\section{Let-7 and miR-29 sensitize NSCLC cells to cisplatin} treatment

Based on the above findings and due to the ability of let7 and miR-29 to act as tumor suppressors, we then examined whether let-7 and miR-29 can directly operate as contributors for cisplatin sensitivity in NSCLC cells. After transfected let-7f or miR-29a mimics into H2030 cells, we detected the cell proliferation by CCK analysis.
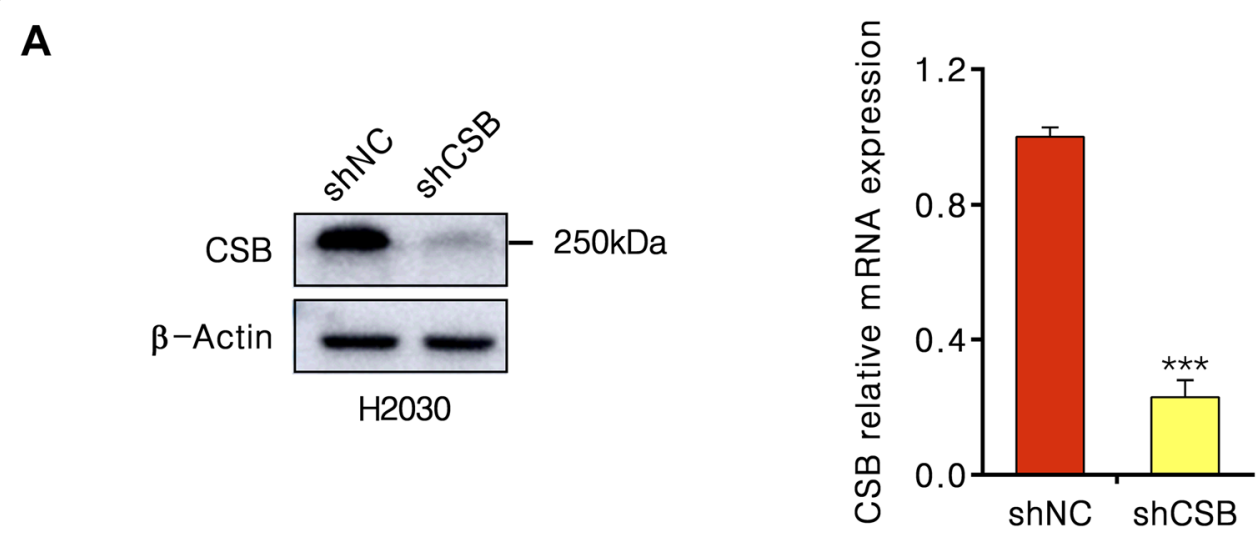

B

C
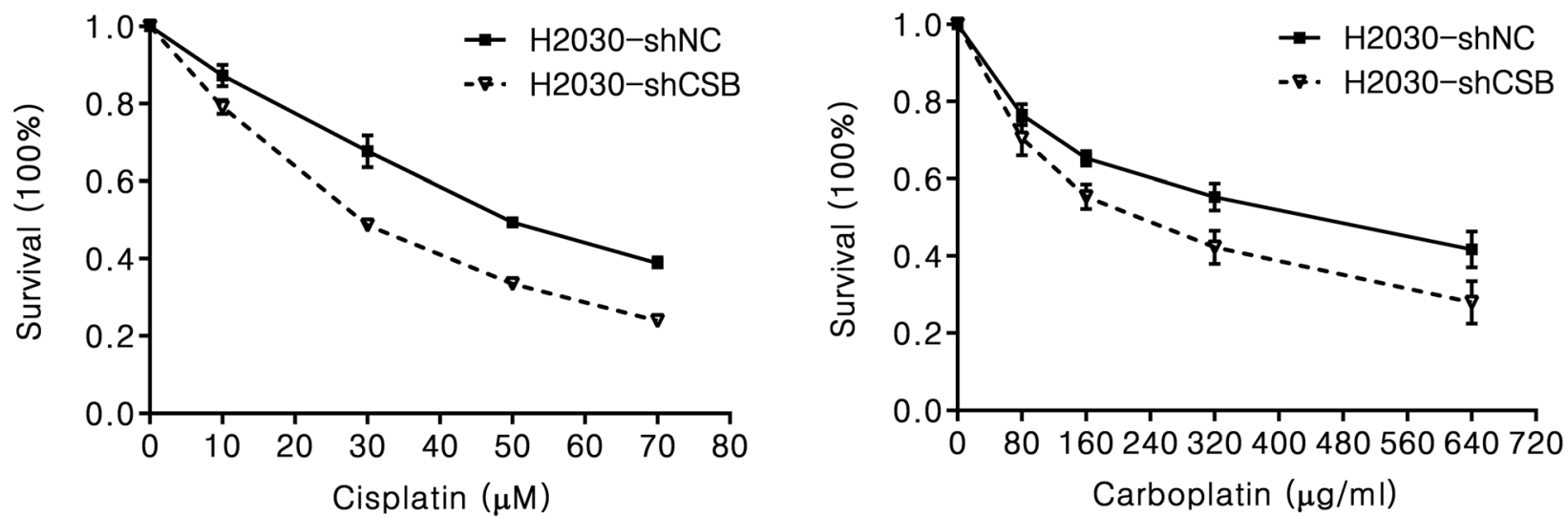

Fig. 6 Short hairpin knockdown of CSB sensitizes H2030 cells to cisplatin and carboplatin. a Immunoblot for CSB in H2030 cells infected with CSB targeting lentiviral shRNAs (a) and corresponding qPCR analysis of relative CSB levels (b). $\mathbf{b}$ and $\mathbf{c}$ Cell growth analysis of H2030-shCSB and H2030-shNC cells with 10-70 $\mu \mathrm{M}$ cisplatin or $80-640 \mu \mathrm{g} / \mathrm{ml}$ carboplatin for $24 \mathrm{~h}$ 


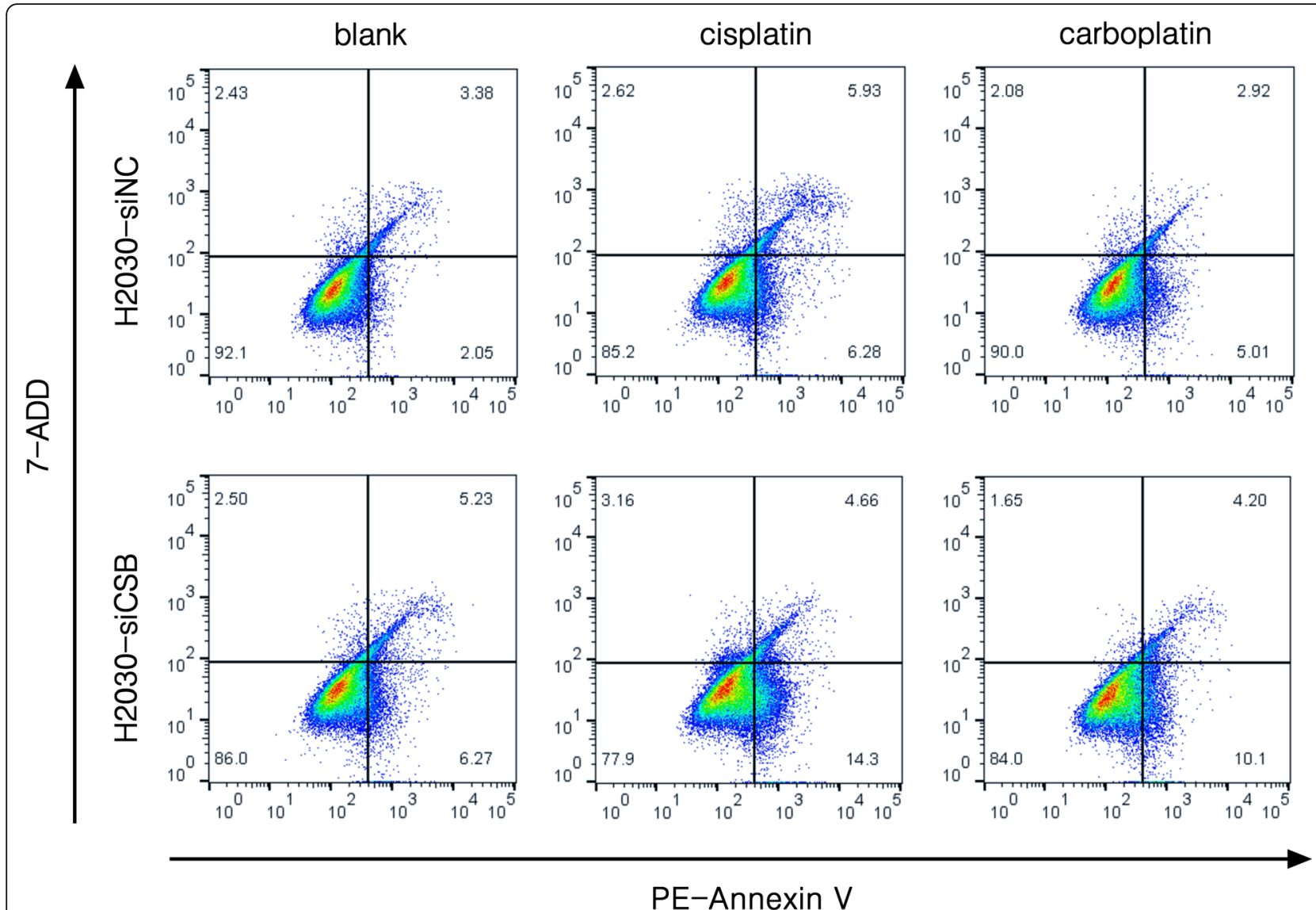

Fig. 7 Inhibition of CSB induces NSCLC cell apoptosis. The effect of CSB siRNA knockdown on apoptosis of H2030 cells treated with $12 \mu \mathrm{M}$ cisplatin or $80 \mu \mathrm{g} / \mathrm{ml}$ carboplatin for $48 \mathrm{~h}$

We observed that both miRNAs effect on the cell proliferation in either cisplatin or carboplatin treated group (Fig. 8). To examine the transcriptional activity of apoptosis target genes, we performed qPCR in let-7f, miR-29a or miR-NC mimics transfected $\mathrm{H} 2030$ cells with cisplatin and carboplatin treatment. The results showed that the RNA levels of a panel of apoptosis target genes have robust changed in either cisplatin/carboplatin treated both miRNAs groups compared to control groups (Fig. 9). The result suggested that both let-7 and miR-29 may directly effect on cisplatin/carboplatin sensitivity in NSCLC.

\section{Discussion}

In this study, we observed CSB is overexpressed in NSCLC regardless of type of histology. We also found that the transcriptional activity of CSB could be suppressed by global decreased let-7 and miR-29 and promoted by additional let-7 or miRNA- 29 in lung cancer cells. After sequestering endogenous let-7 and miR-29 miRNAs, we observed the up-regulation of CSB luciferase activity. The apparent CSB protein occurs in a size of approximately of $250 \mathrm{kDa}$ (CSB monomer of $168 \mathrm{kDa}$ ) in H2030 cells, consistent with the reported model of
CSB wrapping on average 125 bp of DNA around its surface, suggesting strong functional chromatin-remodeling activity of CSB [17].

CSB is a SWI2/SNF2-like DNA-dependent ATPase that can wind DNA [17] and remodel chromatin $[18,19]$. CSB also play important roles in the process of homologous recombination repair (HR) [20, 21], base excision repair (BER) [22, 23], transcription [24] and mitochondrial function [25-27]. Importantly, CSB is overexpressed in a variety of cancer cells including lung cancer and promotes tumor growth, predicting its enhanced repair capacity to cisplatin. Let-7, the well-known tumor suppressor family, is among the most abundantly expressed miRNAs in lung tissue. Global down-regulation of let-7 members is common in lung cancer and has a causative role in the pathogenesis and progression of lung cancer [12].

CSB has been shown to establish a critical negative feedback loop with tumor suppressor p53, which maintains a balance between cellular aging and cancer susceptibility [28]. Interestingly, both let-7 [29-31] and miR-29 [32, 33] seem to form a positive feedback loop with p53 via regulation of upstream regulators of p53 that reinforces its effector functions, such as apoptosis 

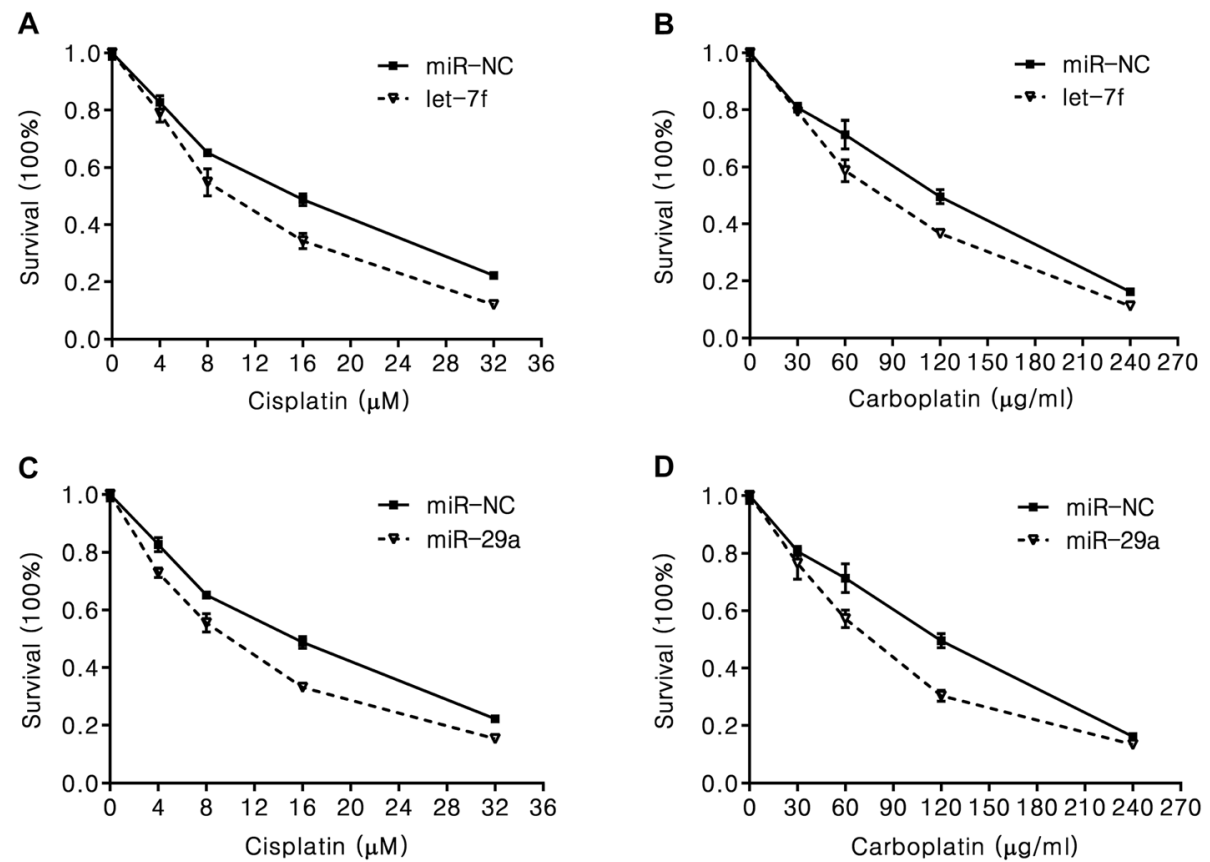

Fig. 8 Let-7 and miR-29 sensitize H2030 cells to cisplatin and carboplatin. a-d Cell proliferation analysis of let-7f and miR-29a or miR-NC transfected H2030 cells with $4-32 \mu \mathrm{M}$ cisplatin or $30-240 \mu \mathrm{g} / \mathrm{ml}$ carboplatin for $48 \mathrm{~h}$

and senescence. Connecting these observations with our results, we presume that let-7 and miR-29-mediated regulation is also a pivotal part of CSB-p53 feedback loop, robustly ensuring the fine CSB-p53 interaction and balance between cellular aging and tumorigenesis.

Our findings also suggested that CSB knockdown could sensitize H2030 cells to platinum-based drugs and induce a potent antiproliferative effect. In consistent with our finding, the suppression of CSB activity has been proved to give rise to cisplatin sensitivity in ovarian, prostate and colon cancer cells $[34,35]$. Recently, two reports have shown that genetic polymorphisms of ERCC6 could affect sensitivity of NSCLC patients to platinum-based chemotherapy, which confirming the important role of CSB in predicting chemotherapy sensitivity and toxicity [36, 37]. Although interstrand crosslinks (ICLs) account for the minority of all types of cisplatin-damages, they are considered extremely toxic by blocking fundamental cellular processes such as replication and transcription and further leading to cell death or genome instability. Researchers also provided many compelling evidences that increased repair of ICLs were highly correlated to the cisplatin resistance [38-40]. CSB plays a critical role in unhooking cisplatin-induced ICLs and restarting transcription in a replication-independent, transcription-
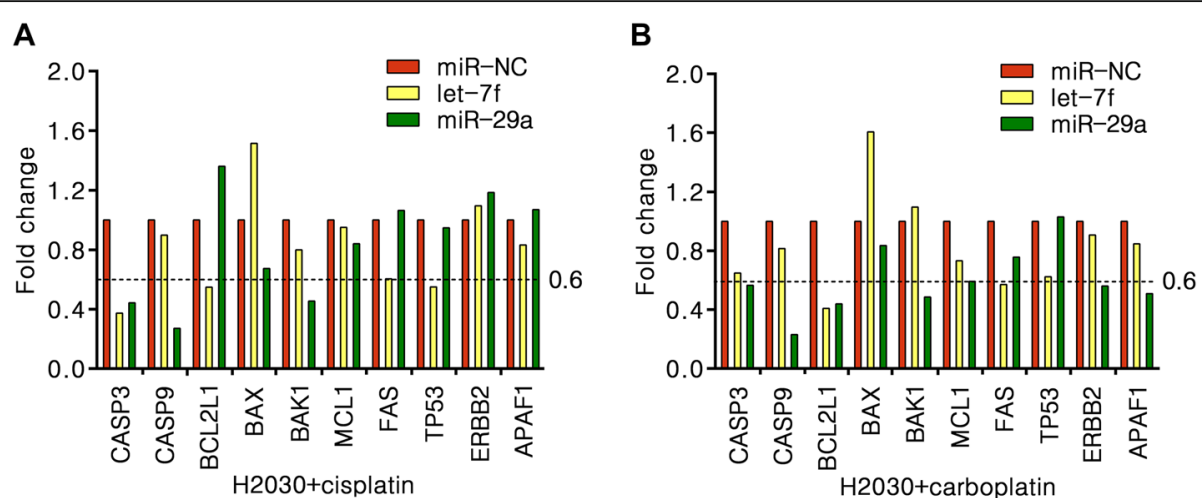

Fig. 9 Let-7 and miR-29 effect on the expression of apoptosis genes in platinum-drug treated NSCLC cells. a H2O30 cells treated with $12 \mu \mathrm{M}$ cisplatin for $48 \mathrm{~h}$. b $\mathrm{H} 2030$ cells treated with $80 \mu \mathrm{g} / \mathrm{ml}$ carboplatin for $48 \mathrm{~h}$ 


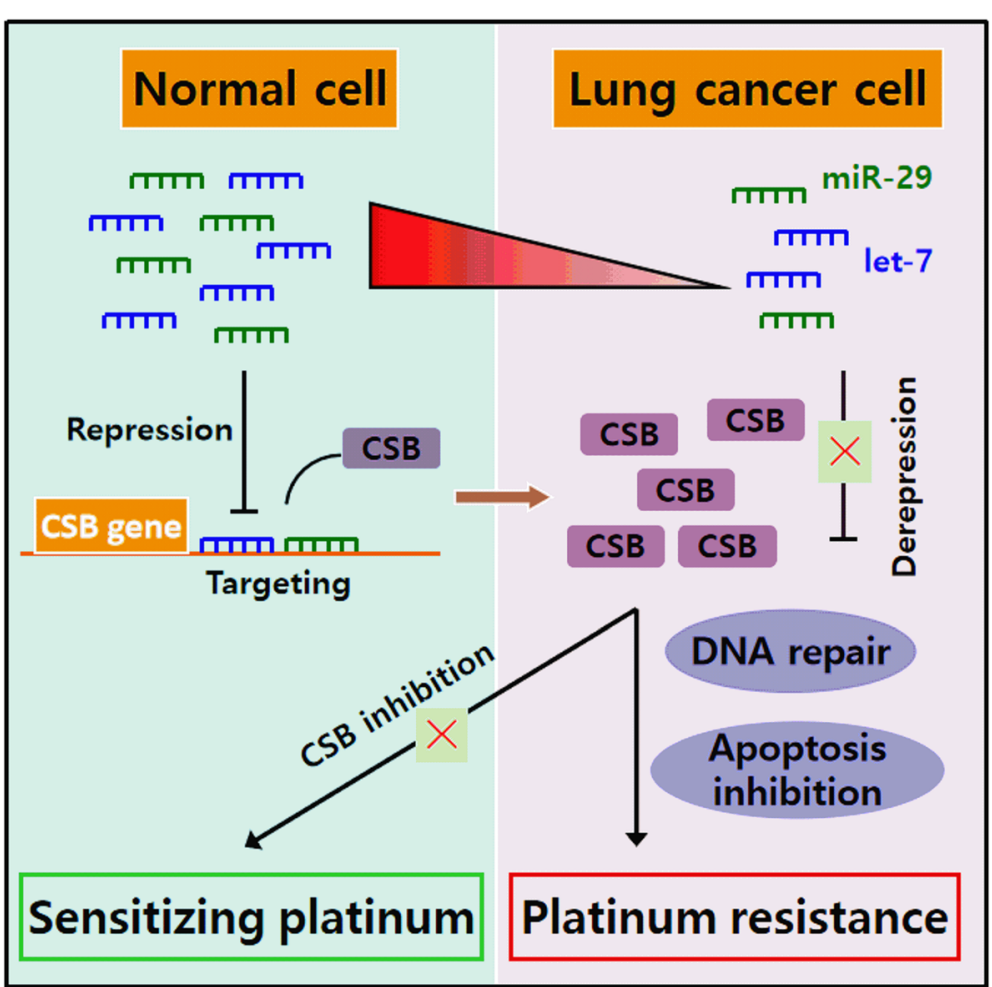

Fig. 10 Schematic diagram summarizing the signaling pathway

associated mechanism, implying its potential as a therapeutic target $[6,11]$. Notably, despite of TCR, CSB also functions in HR and BER pathways specifically, both of which are implicated in the removal of cisplatin induced ICLs [41, 42].

The deregulation of non-coding RNAs, especially miRNA, has emerged as an important mechanism of cisplatin resistance implicated in numerous cancers including lung cancer [43-45]. The ability of a single miRNA to affect the expression of multiple proteins has led to increased interest in miRNAs as mediators of the cellular response to DNA-damaging agents. We found that both let-7 and miR-29 could enhance the sensitivity of cisplatin and carboplatin in H2030 cells with suppression of CSB. This finding is consistent with previous year's reports that let-7 could enhance the sensitivity of glioma, medulloblastoma and esophageal carcinoma to cisplatin treatment and miR-29 modulate the chemosensitivity to cisplatin in nasopharyngeal carcinoma and lung cancer cells [46-50]. Further studies are still required to fully determine whether this effect is common to other NSCLC cells.

\section{Conclusion}

The platinum-based drug resistant of lung cancer cells may involve in the regulation of let-7 and miR-29 to CSB (Fig. 10).

\section{Supplementary information}

Supplementary information accompanies this paper at https://doi.org/10. 1186/s12885-019-6194-Z

Additional file 1. Primers used for the GPCR analysis of mRNAs, related to Figs. 3, 6 and 9 .

\section{Abbreviations}

3'UTR: 3'untranslated region; APA: Alternative polyadenylation; BER: Base excision repair; CSB: Cockayne syndrome protein B; CXCR4: C-X-C motif chemokine receptor 4; DNA: Deoxyribonucleic acid; ERCC1: Excision repair cross-complementation group1; GEPIA: Gene Expression Profiling Interactive Analysis; HR: Homologous recombination repair; ICLs: Interstrand crosslinks; LUAD: Lung adenocarcinoma; LUSC: Lung squamous carcinoma; miRNA: MicroRNA; NC: Negative control; NER: Nucleotide excision repair; NSCLC: Non-small cell lung cancer; PAS: Poly (A) signal; qPCR: Quantitative polymerase chain reaction; TCGA: The Cancer Genome Atlas;

TCR: Transcription-coupled nucleotide excision repair; XPF: Xeroderma pigmentosum group $\mathrm{F}$

\section{Acknowledgments}

Not applicable.

\section{Authors' contributions}

ZY designed, performed experiments and drafted the manuscript. $\mathrm{CL}$ performed immunohistochemisty. HW, YX and HG assisted with luciferase reporter analysis. XZ was involved in research design, data interpretation, supervision of the project and revising the manuscript. All authors read and approved the final manuscript.

\section{Funding}

This study was supported by the National Natural Science Foundation of China (grant numbers 81272613), Hebei high-level talent cultivation plan in University (grant numbers GCC2014050) and Key Project of Natural 
Science Foundation of Hebei province of China (grant numbers H2017209233). The funding bodies did not have any role in the design of the study and collection, analysis and interpretation of data and in writing of the manuscript.

\section{Availability of data and materials}

The datasets used and/or analyzed during the current study are available from the corresponding author on reasonable request.

\section{Ethics approval and consent to participate}

This study was approved by the ethics committee of North China University of Science and Technology (No. 12-002) and written informed consent was obtained from all patients. Details can be provided by the corresponding author on reasonable request.

\section{Consent for publication}

Not applicable.

\section{Competing interests}

The authors declare that they have no competing interests.

\section{Author details}

${ }^{1}$ Institute of Molecular Genetics, College of Life Science, North China University of Science and Technology, Tangshan, China. ${ }^{2}$ Hebei Key Laboratory of Basic Medicine for Chronic Disease, School of Basic Medical Sciences, North China University of Science and Technology, Tangshan, China. ${ }^{3}$ Department of Pathology, Affiliated Tangshan Renmin Hospital North China University of Science and Technology, Tangshan, China. ${ }^{4}$ Institute of Epidemiology, School of Public Health, North China University of Science and Technology, Tangshan, China.

\section{Received: 11 March 2019 Accepted: 24 September 2019}

\section{Published online: 15 October 2019}

\section{References}

1. Housman G, Byler S, Heerboth S, Lapinska K, Longacre M, Snyder N, et al. Drug resistance in cancer: an overview. Cancers. 2014;6(3):1769-92.

2. Ferreira JA, Peixoto A, Neves M, Gaiteiro C, Reis CA, Assaraf YG, et al. Mechanisms of cisplatin resistance and targeting of cancer stem cells: adding glycosylation to the equation. Drug Resist Update. 2016;24:34-54.

3. Bach D-H, Hong J-Y, Park HJ, Lee SK. The role of exosomes and miRNAs in drug-resistance of cancer cells. Int J Cancer. 2017;141(2):220-30.

4. Jung YW, Lippard SJ. Direct cellular responses to platinum-induced DNA damage. Chem Rev. 2007;107(5):1387-407.

5. Reardon JT, Vaisman A, Chaney SG, Sancar A. Efficient nucleotide excision repair of cisplatin, oxaliplatin, and bis-aceto-ammine-dichlorocyclohexylamine-platinum(IV) (JM216) platinum intrastrand DNA diadducts. Cancer Res. 1999:59(16):3968-71.

6. Enoiu M, Jiricny J, Scharer OD. Repair of cisplatin-induced DNA interstrand crosslinks by a replication-independent pathway involving transcriptioncoupled repair and translesion synthesis. Nucleic Acids Res. 2012;40(18): 8953-64.

7. Marteijn JA, Lans H, Vermeulen W, Hoeijmakers JHJ. Understanding nucleotide excision repair and its roles in cancer and ageing. Nat Rev Mol Cell Bio. 2014;15(7):465-81.

8. Bowden NA. Nucleotide excision repair: why is it not used to predict response to platinum-based chemotherapy? Cancer Lett. 2014;346(2): 163-71.

9. Arora S, Kothandapani A, Tillison K, Kalman-Maltese V, Patrick SM. Downregulation of XPF-ERCC1 enhances cisplatin efficacy in cancer cells. DNA Repair. 2010;9(7):745-53.

10. Caputo M, Frontini M, Velez-Cruz R, Nicolai S, Prantera G, Proietti-De-Santis $L$. The CSB repair factor is overexpressed in cancer cells, increases apoptotic resistance, and promotes tumor growth. DNA Repair. 2013;12(4):293-9.

11. lyama T, Lee SY, Berquist BR, Gileadi O, Bohr VA, Seidman MM, et al. CSB interacts with SNM1A and promotes DNA interstrand crosslink processing. Nucleic Acids Res. 2015;43(1):247-58.

12. Osada H, Takahashi T. Let-7 and miR-17-92: small-sized major players in lung cancer development. Cancer Sci. 2011;102(1):9-17.
13. Fabbri M, Garzon R, Cimmino A, Liu Z, Zanesi N, Callegari E, et al. MicroRNA29 family reverts aberrant methylation in lung cancer by targeting DNA methyltransferases 3A and 3B. P Natl Acad Sci USA. 2007;104(40):15805-10.

14. Agarwal V, Bell GW, Nam JW, Bartel DP. Predicting effective microRNA target sites in mammalian mRNAs. Elife. 2015;4:e05005.

15. Troelstra C, Hesen W, Bootsma D, Hoeijmakers JHJ. Structure and expression of the excision repair gene Ercc6, involved in the human disorder Cockaynes-syndrome group-B. Nucleic Acids Res. 1993;21(3):419-26.

16. Johnson CD, Esquela-Kerscher A, Stefani G, Byrom N, Kelnar K, Ovcharenko $D$, et al. The let-7 MicroRNA represses cell proliferation pathways in human cells. Cancer Res. 2007;67(16):7713-22.

17. Beerens N, Hoeijmakers JHJ, Kanaar R, Vermeulen W, Wyman C. The CSB protein actively wraps DNA. J Biol Chem. 2005;280(6):4722-9.

18. Citterio E, Van Den Boom V, Schnitzler G, Kanaar R, Bonte E, Kingston RE, et al. ATP-dependent chromatin remodeling by the Cockayne syndrome $B$ DNA repair-transcription-coupling factor. Mol Cell Biol. 2000;20(20):7643-53.

19. Newman JC, Bailey AD, Weiner AM. Cockayne syndrome group B protein (CSB) plays a general role in chromatin maintenance and remodeling. P Natl Acad Sci USA. 2006:103(25):9613-8.

20. Batenburg NL, Thompson EL, Hendrickson EA, Zhu XD. Cockayne syndrome group B protein regulates DNA double-strand break repair and checkpoint activation. EMBO J. 2015;34(10):1399-416.

21. Wei LZ, Nakajima S, Bohm S, Bernstein KA, Shen ZY, Tsang M, et al. DNA damage during the G0/G1 phase triggers RNA-templated, Cockayne syndrome B-dependent homologous recombination. P Natl Acad Sci USA. 2015;112(27):E3495-E504

22. Stevnsner T, Muftuoglu M, Aamann MD, Bohr VA. The role of Cockayne syndrome group B (CSB) protein in base excision repair and aging. Mech Ageing Dev. 2008;129(7-8):441-8.

23. Aamann MD, Hvitby C, Popuri V, Muftuoglu M, Lemminger L, Skeby CK, et al. Cockayne syndrome group B protein stimulates NEIL2 DNA glycosylase activity. Mech Ageing Dev. 2014;135:1-14.

24. Velez-Cruz R, Egly JM. Cockayne syndrome group B (CSB) protein: at the crossroads of transcriptional networks. Mech Ageing Dev. 2013;134(5-6):234-42.

25. Aamann MD, Sorensen MM, Hvitby C, Berquist BR, Muftuoglu M, Tian JY, et al. Cockayne syndrome group B protein promotes mitochondrial DNA stability by supporting the DNA repair association with the mitochondrial membrane. FASEB J. 2010:24(7):2334-46.

26. Berquist BR, Canugovi C, Sykora P, Wilson DM, Bohr VA. Human Cockayne syndrome B protein reciprocally communicates with mitochondrial proteins and promotes transcriptional elongation. Nucleic Acids Res. 2012;40(17):8392-405.

27. Cleaver JE, Brennan-Minnella AM, Swanson RA, Fong KW, Chen JJ, Chou KM, et al. Mitochondrial reactive oxygen species are scavenged by Cockayne syndrome B protein in human fibroblasts without nuclear DNA damage. P Natl Acad Sci USA. 2014;111(37):13487-92.

28. Frontini $M$, Proietti-De-Santis L. Interaction between the Cockayne syndrome B and p53 proteins: implications for aging. Aging-Us. 2012:4(2):89-97.

29. Hermeking H, Eick D. Mediation of C-Myc-induced apoptosis by P53. Science. 1994:265(5181):2091-3.

30. Chang TC, Yu DN, Lee YS, Wentzel EA, Arking DE, West KM, et al. Widespread microRNA repression by Myc contributes to tumorigenesis. Nat Genet. 2008;40(1):43-50.

31. Nguyen DTT, Richter D, Michel G, Mitschka S, Kolanus W, Cuevas E, et al. The ubiquitin ligase LIN41/TRIM71 targets p53 to antagonize cell death and differentiation pathways during stem cell differentiation. Cell Death Differ. 2017;24(6):1063-78.

32. Park SY, Lee JH, Ha M, Nam JW, Kim VN. miR-29 miRNAs activate $p 53$ by targeting p85a and CDC42. Nat Struct Mol Biol. 2009;16(1):23-9.

33. Ugalde AP, Ramsay AJ, de la Rosa J, Varela I, Marino G, Cadinanos J, et al. Aging and chronic DNA damage response activate a regulatory pathway involving miR-29 and p53. EMBO J. 2011;30(11):2219-32.

34. Lu Y, Mani S, Kandimalla ER, Yu D, Agrawal S, States JC, et al. The Cockayne syndrome group B DNA repair protein as an anti-cancer target. Int J Oncol. 2001:19(6):1089-97.

35. Stubbert L, Smith JM, McKay BC. Decreased transcription-coupled nucleotide excision repair capacity is associated with increased p53-and MLH1independent apoptosis in response to cisplatin. BMC Cancer. 2010;10:207.

36. Song $X$, Wang $S M$, Hong $X$, Li XY, Zhao XY, Huai $C$, et al. Single nucleotide polymorphisms of nucleotide excision repair pathway are significantly associated with outcomes of platinum-based chemotherapy in lung cancer. Sci Rep-Uk. 2017;7:5082. 
37. Cui JJ, Wang LY, Zhu T, Gong WJ, Zhou HH, Liu ZQ, et al. Gene-gene and gene-environment interactions influence platinumbased chemotherapy response and toxicity in non-small cell lung cancer patients. Sci Rep-Uk. 2017;7:11785.

38. Zhen WP, Link CJ, Oconnor PM, Reed E, Parker R, Howell SB, et al. Increased gene-specific repair of cisplatin interstrand cross-links in cisplatin-resistant human ovarian-cancer cell-lines. Mol Cell Biol. 1992;12(9):3689-98.

39. Petersen LN, Mamenta EL, Stevnsner T, Chaney SG, Bohr VA. Increased gene specific repair of cisplatin induced interstrand crosslinks in cisplatin resistant cell lines, and studies on carrier ligand specificity. Carcinogenesis. 1996; 17(12):2597-602.

40. Masumoto N, Nakano S, Fujishima H, Kohno K, Niho Y. v-src induces cisplatin resistance by increasing the repair of cisplatin-DNA interstrand cross-links in human gallbladder adenocarcinoma cells. Int J Cancer. 1999; 80(5):731-7.

41. Macerelli M, Ganzinelli M, Gouedard C, Broggini M, Garassino MC, Linardou H, et al. Can the response to a platinum-based therapy be predicted by the DNA repair status in non-small cell lung cancer? Cancer Treat Rev. 2016;48:8-19.

42. Sawant A, Floyd AM, Dangeti M, Lei W, Sobol RW, Patrick SM. Differential role of base excision repair proteins in mediating cisplatin cytotoxicity. DNA Repair. 2017:51:46-59.

43. Donzelli S, Fontemaggi G, Fazi F, Di Agostino S, Padula F, Biagioni F, et al. MicroRNA-128-2 targets the transcriptional repressor E2F5 enhancing mutant p53 gain of function. Cell Death Differ. 2012;19(6):1038-48.

44. Dong Z, Zhong ZW, Yang LH, Wang SM, Gong ZH. MicroRNA-31 inhibits cisplatin-induced apoptosis in non-small cell lung cancer cells by regulating the drug transporter ABCB9. Cancer Lett. 2014;343(2):249-57.

45. Cheng MW, Shen ZT, Hu GY, Luo LG. Prognostic significance of microRNA-7 and its roles in the regulation of cisplatin resistance in lung adenocarcinoma. Cell Physiol Biochem. 2017;42(2):660-72.

46. Xie C, Chen W, Zhang M, Cai Q, Xu W, Li X, et al. MDM4 regulation by the let-7 miRNA family in the DNA damage response of glioma cells. FEBS Lett. 2015;589(15):1958-65.

47. Pannuru P, Dontula R, Khan AA, Herbert E, Ozer H, Chetty C, et al. miR-let7f-1 regulates SPARC mediated cisplatin resistance in medulloblastoma cells. Cell Signal. 2014;26(10):2193-201.

48. Sugimura K, Miyata $\mathrm{H}$, Tanaka K, Hamano R, Takahashi T, Kurokawa Y, et al. Let-7 expression is a significant determinant of response to chemotherapy through the regulation of IL-6/STAT3 pathway in esophageal squamous cell carcinoma. Clin Cancer Res. 2012;18(18):5144-53.

49. Wu Y, Crawford M, Mao Y, Lee RJ, Davis IC, Elton TS, et al. Therapeutic delivery of microRNA-29b by cationic lipoplexes for lung cancer. Mol Ther Nucleic Acids. 2013;2:e84.

50. Zhang J-X, Qian D, Wang F-W, Liao D-Z, Wei J-H, Tong Z-T, et al. MicroRNA-29C enhances the sensitivities of human nasopharyngeal carcinoma to cisplatinbased chemotherapy and radiotherapy. Cancer Lett. 2013;329(1):91-8.

\section{Publisher's Note}

Springer Nature remains neutral with regard to jurisdictional claims in published maps and institutional affiliations.

Ready to submit your research? Choose BMC and benefit from:

- fast, convenient online submission

- thorough peer review by experienced researchers in your field

- rapid publication on acceptance

- support for research data, including large and complex data types

- gold Open Access which fosters wider collaboration and increased citations

- maximum visibility for your research: over $100 \mathrm{M}$ website views per year

At BMC, research is always in progress.

Learn more biomedcentral.com/submissions 